\title{
INCREASED IMPULSIVE BEHAVIOR AND RISK PRONENESS FOLLOWING LENTIVIRUS-MEDIATED DOPAMINE TRANSPORTER OVER-EXPRESSION IN RATS' NUCLEUS ACCUMBENS
}

\author{
W. ADRIANI, ${ }^{\text {a1 }}$ F. BOYER, ${ }^{\text {b1 }}$ L. GIOIOSA, ${ }^{\text {a }}$ S. MACRÌ, ${ }^{a}$ \\ J.-L. DREYER ${ }^{\mathrm{b}}$ AND G. LAVIOLA ${ }^{\mathrm{a} *}$ \\ ${ }^{a}$ Behavioral Neuroscience Section, Department of Cell Biology and \\ Neurosciences, Istituto Superiore di Sanità, viale Regina Elena 299, \\ I-00161, Roma, Italy \\ ${ }^{b}$ Department of Medicine, University of Fribourg, Switzerland
}

\begin{abstract}
Multiple theories have been proposed for sensation seeking and vulnerability to impulse-control disorders [Zuckerman M, KuhIman DM (2000) Personality and risk-taking: Common biosocial factors. J Pers 68:999-1029], and many of these rely on a dopamine system deficit. Available animal models reproduce only some behavioral symptoms and seem devoid of construct validity. We used lentivirus tools for over-expressing or silencing the dopamine transporter (DAT) and we evaluated the resulting behavioral profiles in terms of motivation and self-control. Wistar adult rats received stereotaxic inoculation of a lentivirus that allowed localized intra-accumbens delivery of a DAT gene enhancer/ silencer, or the green fluorescent protein, GFP. These animals were studied for intolerance to delay, risk proneness and novelty seeking. As expected, controls shifted their demanding from a large reward toward a small one when the delivery of the former was increasingly delayed (or uncertain). Interestingly, in the absence of general locomotor effects, DAT over-expressing rats showed increased impulsivity (i.e. a more marked shift of demanding from the large/ delayed toward the small/soon reward), and increased risk proneness (i.e. a less marked shift from the large/uncertain toward the small/sure reward), compared with controls. Rats with enhanced or silenced DAT expression did not show any significant preference for a novel environment. In summary, consistent with literature on comorbidity between attentiondeficit/hyperactivity disorder and pathological gambling, we demonstrate that DAT over-expression in rats' nucleus accumbens leads to impulsive and risk prone phenotype. Thus, a reduced dopaminergic tone following altered accumbal DAT function may subserve a sensation-seeker phenotype and the vulnerability to impulse-control disorders. (C) 2009 IBRO. Published by Elsevier Ltd. All rights reserved.
\end{abstract}

Key words: dopamine transporter, sensation seeking, intolerance to delay, probabilistic delivery.

\footnotetext{
${ }^{1}$ Both authors contributed equally to this work.

${ }^{*}$ Corresponding author. Tel: +39-06-4990-2105; fax: +39-06-495-7821. E-mail address: laviola@iss.it (G. Laviola).

Abbreviations: ADHD, attention-deficit/hyperactivity disorder; DA, dopamine; DAT, dopamine transporter; GFP, green fluorescent protein; LL, large/late; LLL, large/luck-linked; NAcc, nucleus accumbens; NOP, non-operated; PG, pathological gambling; SIL, silenced; SS, small/ soon or small/sure; TBST, $10 \mathrm{mM}$ Tris, $150 \mathrm{mM} \mathrm{NaCl}$, and $0.1 \%$ Tween-20.
}

Sensation seeking is a supertrait expressed by some individuals who are often involved in very extreme behaviors and risky activities, such as exaggerate sexual behavior, reckless driving, drug misuse, and pathological gambling (PG) (Zuckerman and Kuhlman 2000). Noteworthy, there is substantial genetic influence in these sensation-seeking traits: for instance, the dopamine (DA) D4 receptor is the prototypic polymorphic gene subserving a background for novelty seeking (Ebstein et al., 1996), drug abuse (Laucht et al., 2005) and vulnerability to attention-deficit hyperactivity disorder (ADHD) (Faraone et al., 2005) and PG (Comings et al., 1999).

ADHD is heterogeneous, is highly heritable, and results from complex gene-gene and gene-environment interactions. ADHD affects $1-4 \%$ of children, representing a social burden (Biederman, 1998). Beside core symptoms of hyperactivity, impulsivity and impaired sustained attention, which are also found in other syndromes, ADHD children often display accompanying behavioral difficulties, including a disinhibited conduct and obsessive-compulsive symptoms (Snyder et al., 2002). According to the dominant model, ADHD is viewed as an executive dysfunction (Doyle, 2006; Castellanos et al., 2006; Willcutt et al., 2005), but alternative accounts present ADHD as a motivational dysfunction (Sonuga-Barke, 2005), arising from altered reward processes within fronto-striatal circuits (Sagvolden and Sergeant, 1998; Oades, 1998). Very frequently comorbid with ADHD (Sood et al., 2003), PG is a chronic, progressive disorder with a prevalence of $1-4 \%$, and is rapidly emerging as a mental health concern among Western civilizations. PG is essentially an impulse-control disorder, commonly comorbid to compulsive buying and compulsive sexual behavior (Black and Moyer, 1998). PG may also be conceptualized as an addictive disorder and/or as part of the obsessive-compulsive spectrum (Lowengrub et al., 2006): in fact, more than half of PG patients have an obsessive-compulsive, schizotypal and paranoid personality, as well as substance abuse/dependence problems (Hollander et al., 2000, 2005).

The deficits in cognitive control and/or motivation seen in sensation seeking, ADHD and PG highlight the importance of DA and 5-HT systems' disruption in these disorders. Indeed, 5-HT subserves (dis)inhibition via cortical control over behavioral initiation, which is important for the difficulty in controlling instinctive reactions and temptations (Hollander et al., 2000). DA systems are involved in many reinforcement-related processes: 1 ) they subserve motivation to sustain effort toward positive (attracting) and negative (avoided) stimuli, and may subserve the addictive/ 
compulsive component of these disorders (Hollander et al., 2000); 2) they are involved in forming predictions about future outcomes, in that DA neuronal firing is linked to detecting discrepancies between actual and expected outcomes (Schultz et al., 1997). Learning when, or in which contexts, to expect what, is a critical event for adjusting behavior appropriately (Casey and Durston, 2006) when predicted outcomes are violated. Likewise, even if the ability to approach/avoid salient stimuli and/or to predict/ detect (ir)regularities in the environment is intact, any inefficient top-down inhibitory control could result in poor feedback regulation of behavior. The variability of ADHD sub-populations reported in the medical literature may be partly due to differences in the relative dysfunction between DA and 5-HT systems (Sagvolden and Sergeant, 1998; Oades 1998).

These two systems are indeed strong candidates for pathogenesis of sensation seeking, ADHD and PG, but there is not a universally valid animal model yet (Russell et al., 2005; Sagvolden et al., 2005). Current animal models mimic distinct behavioral characteristics of these disorders, bearing different neural defects, and are mostly genetic, like the spontaneously hypertensive rats (SHR), the dopamine transporter (DAT) knockout mice, the synaptosomal-associated protein $25 \mathrm{kDa}$ mutant mice, or mice expressing a mutant thyroid receptor. The validity of these models is however limited, due to lack of construct validity, thus promoting the efforts to identify novel animal models. Many studies have described modifications on DAT expression at least in ADHD (Fischman et al., 1998; Dougherty et al., 1999; Jucaite et al., 2005; Bannon 2005). We have tested the efficacy of lentiviral tools, driving the expression of DAT or siRNAs targeted against DAT mRNA in rats. It is well known that DAT gene is expressed in the midbrain (A9 and A10 areas), and proteins are then transported into the dorsal striatum and nucleus accumbens (NAcc). The aim of this study was to investigate behavioral effects of over-expression and/or down-regulation of DAT in this specific brain area, driving motivated behavior and impulsivity, rather than in the whole dopaminergic system. As a matter of fact, it is well established that DAT mRNA can be found in the NAcc (Maggos et al., 1997). Moreover, the capacity of lentiviruses to be retro-transported is well established (Szulc et al., 2006). We have demonstrated previously that lentivirus-transfected genes undergo a retrograde transport from the NAcc (Boyer and Dreyer, 2008), thus enabling DAT gene and siRNA expression within the ventral tegmental area (VTA). The in vivo efficacy of our DAT enhancer and of the three silencing siRNAs has been demonstrated as well (Boyer and Dreyer, 2008). DAT protein product is then transported and expressed within the NAcc (Boyer and Dreyer, 2008). Such an approach enabled the evaluation of behavioral changes, associated with focal meso-limbic DAT over-expression and/or suppression (Mazei-Robinson and Blakely, 2006).

We investigated here the relevant behavioral symptoms produced by enduring alteration of DAT function (Madras et al., 2005; Spencer et al., 2005; Thapar et al., 2005). Accumbal DAT suppression was expected to en- hance tonic DA transmission, compared with controls, whereas its over-expression would drastically reduce synaptic DA levels. The NAcc was selected as the inoculation site since its lesions are known to cause hyperactivity and impulsive choice in rats (Cardinal et al., 2004). Our aim was to establish whether modulation of genetic DAT levels would lead rats to exhibit a deficit in motivational and/or impulse-control endpoints. Thus, to probe the resulting phenotype, animals were tested for their drive to seek for novelty, intolerance to delay, and temptation to gamble (Laviola et al., 2003; Adriani and Laviola, 2006). We demonstrate here that a peculiar behavioral profile was specific of DAT + rats. To investigate the molecular correlates of such profile, NAcc samples, collected at sacrifice of DAT+ and control animals, were processed for DAT gene expression and protein density.

\section{EXPERIMENTAL PROCEDURES}

Animal experimental protocols were approved by institutional Animal Survey Board, on behalf of Ministry of Health, and were in close agreement with European Community Directives and Italian law. All efforts were made to minimize animal suffering, to reduce the number of animals used, and to use alternatives to in vivo testing.

\section{Subjects, breeding, and rearing conditions}

Upon arrival, Wistar male rats (Harlan, Correzzana, Italy) weighing 200-250 g were housed in pairs, inside Plexiglas Macrolon III cages with metal tops and a sawdust bedding. Rats were located in an air-conditioned room (temperature $21 \pm 1{ }^{\circ} \mathrm{C}$, relative humidity $60 \pm 10 \%$ ), with a 12-h light/dark cycle (lights off from 21:00-09:00 h). Water and food (Enriched Standard Diet, Mucedola, Settimo Milanese, Italy) were available ad libitum. After 2 weeks of acclimation, animals were inoculated with lentiviral vectors for gene transfer. After 2 weeks of post-surgical recovery, they were tested with the impulsivity (i.e. delay-intolerance) and risk-proneness (i.e. probabilistic-delivery) operant tasks and for novelty seeking.

\section{Construction of Lenti-DAT, stereotaxic surgery}

The DAT cDNA (GenBank accession no. 012694) was amplified by reverse transcription. The cDNA was then PCR amplified from pCMV5rDAT as a template and the following primers: $5^{\prime}-C C G$ TTA ACA TGG ACT ACA AAG ACG ATG ACG ATA AGC CAG TAA GAG CAA ATG C-3' and 5'-CCG CTC GAG CGG TTA CAG CAA CAG CCA GTG ACG-3'. The forward primer contains an Hpal restriction site followed by a FLAG epitope sequence (in bold) and the rat $5^{\prime}$ DAT cDNA specific sequence, the reverse primer contains the rat $3^{\prime}$ DAT cDNA specific sequence, a stop codon and a Xhol restriction site. The PCR product was digested with $\mathrm{Hpal}$ and $\mathrm{Xhol}$ and cloned into $\mathrm{Hpal} / \mathrm{Xhol}$ restriction sites into pTK431 (Bahi et al., 2005a,b), which expresses the gene of interest under control of a Doxy-Off promoter: namely, doxycycline can switch off the Lenti-DAT-induced over-expression. To silence the DAT expression in vivo, three targets were designed according to the DAT mRNA sequence, and similarly cloned into pTK431. The following targets within the DAT sequence were selected: 1st target, bp 19-47; 2nd target, bp 864-890; 3rd target, bp 18271854. This selection (http://katahdin.cshl.org:9331/RNAi/html/ rnai.html) was based on the Hannon's design criterion. To each oligo, a Xhol restriction site was added at $3^{\prime}$ and a U6-3'-specific 10 mer at $5^{\prime}$. Using the pSilencer 1.0-U6 (Ambion, UK) as a template and a U6 promoter-specific forward primer containing BamHI restriction site (5'-GCG GAT CCC GCT CTA GAA CTA GTG $C-3^{\prime}$ ), each siRNA target was added to the mouse U6 
promoter, using the following PCR program: $120 \mathrm{~s}$ at $94{ }^{\circ} \mathrm{C}$ (initial denaturation) followed by 35 cycles $\left(45 \mathrm{~s}\right.$ at $94^{\circ} \mathrm{C}, 45 \mathrm{~s}$ at $64^{\circ} \mathrm{C}$ and $45 \mathrm{~s}$ at $72{ }^{\circ} \mathrm{C}$ ) in $4 \%$ dimethyl sulfoxide (Sigma, Switzerland). The PCR product was digested with $\mathrm{BamHI}$ and $\mathrm{Xhol}$, cloned into similar sites into pTK431, and sequenced to verify the integrity of each construct. The efficacy of these siRNAs has been demonstrated previously (Boyer and Dreyer, 2008).

The green fluorescent protein (GFP) expression vector pTK433 (Bahi et al., 2004a,b) was used as a control vector. The vector plasmids (pTK431-DAT, pTK431-DAT-siRNAs, or pTK433GFP), together with the packaging construct plasmid $p \Delta N R F$ and the envelope plasmid pMDG-VSV-G, were co-transfected into HEK293T cells to produce the viral particles (Bahi et al., 2004a,b). The viral titers were determined. For the in vivo experiments, the different viral stocks were matched for viral particle content and will be used at $0.2 \mathrm{mg} / \mathrm{mL}$ of p24 antigen, a direct measure of the virus concentration (Boyer and Dreyer, 2008). The efficiency of the Lenti-DAT was tested in vitro by infection of HEK293T cells. Animals were bilaterally injected into the NAcc (anterior +1.4 , lateral \pm 1.2 , ventral -7.8 from bregma, Paxinos and Watson, $1998)$ with concentrated lentiviral stock $(0.2 \mathrm{mg} / \mathrm{mL}$ of $\mathrm{p} 24$, corresponding to $8 \times 109 \mathrm{IU} / \mathrm{mL}$ ): $2 \mu \mathrm{L}$ of Lenti-DAT alone (enhancer, DAT), $2 \mu \mathrm{L}$ of Lenti-DAT-siRNAs (silenced, SIL), or $2 \mu \mathrm{L}$ LentiGFP (control). A non-operated (NOP) group of rats was also included in the experimental design and in the preliminary analyses.

\section{Experiment 1: Novelty-preference task}

The experimental apparatus consisted of an opaque-Plexiglas box with smooth walls and floor $(70 \times 30 \times 35 \mathrm{~cm})$, subdivided into two chambers separated by a partition possessing a doorway through which rats could traverse. One chamber had a white floor, the other one had a black floor, but both chambers had gray walls. Animal locomotion were recorded by four photocells per chamber, placed at few $\mathrm{cm}$ from the floor, and connected to an IBM computer equipped with custom-made software. The following measures were obtained automatically: 1) time spent in each compartment; and 2) activity rate in each compartment.

The whole experimental schedule took a total of 4 days, each subject from each of the four groups being tested between 10:00 and 18:00 h, with testing of different groups being counterbalanced across time. During the Familiarization phase (days 1-3), animals were gently placed for $40 \mathrm{~min}$ in one compartment of the apparatus. During the Novelty-Preference task (on day 4), animals were placed in the familiar compartment for a 5-min session. The partition separating the two compartments of the apparatus was then removed, and rats were thus allowed to freely explore the whole apparatus (both the familiar and the novel sides) for $35 \mathrm{~min}$. The test was carried out under dim illumination and the floor of the apparatus was cleaned after each animal testing.

\section{Experiment 2: Delay-intolerance task for impulsivity}

Animals were tested by the intolerance-to-delay protocol, involving a choice between a small, immediate reinforcer and a larger, delayed one. For a few days before the schedule started, animals were food-restricted to keep them at $80-85 \%$ of their free-feeding weight, in order to increase their motivation to work for food delivery. Despite this slight food restriction, the animals were fully healthy and expressed a normal repertoire of spontaneous behavior in the home cage (informal observations in the animal room). Each animal was then placed daily in a computer-controlled operant chamber (Coulbourn Instruments, Allentown, USA), provided with two nose-poking holes, two chamber lights, two feeder devices, two magazines where precision pellets $(45 \mathrm{mg}$, BioServ, Frenchtown, USA) were dropped, and two magazine lights. The nose-poking in either hole was detected by a photocell and was recorded by a computer, which also controlled food delivery. After the 25-min session, animals were returned to their home cage, where they were given standard chow (approximately $10 \mathrm{~g} / \mathrm{each}$ ).

During the training phase (1 week), nose-poking in one of the two holes resulted in the delivery of five pellets of food in one magazine, whereas nose-poking in the other hole resulted in only one pellet of food in the other magazine. After nose-poking and before food delivery, the chamber light over the appropriate magazine was turned on for $1 \mathrm{~s}$. Following food delivery, the appropriate magazine light was turned on for $20 \mathrm{~s}$, during which nose-poking was recorded but was without scheduled consequences (time-out). During the testing phase (1 week), a signaled delay was inserted between nose-poking and large-reward delivery. During such a delay interval, any additional nose-poking was recorded but had no scheduled consequences ("inadequate responding"). Hence, animals had to make a choice between a large/late (LL) or a small/soon (SS) reward. The delay was signaled by chamber light (over the largereward magazine) being kept on during the entire delay length. For large-reward delivery, the delay length was fixed for each daily session, and was changed progressively over subsequent days. The small-reward delivery was unchanged.

\section{Experiment 3: Probabilistic-delivery task for risk proneness}

Animals were tested by the probabilistic-delivery protocol, involving a choice between a small, certain reinforcer and a larger, uncertain one. Feeding schedule and operant chambers were the same as the previous task. After a 20 -min session, animals were returned to their home cage and given standard chow (approximately $10 \mathrm{~g} / \mathrm{each}$ ).

Nose-poking in one of the two holes always resulted in the delivery of one pellet of food, whereas nose-poking in the other hole could result (or not) in five pellets of food. Both rewards were delivered in the same (feeding) magazine, but the large-reward delivery could be released or omitted according to a given percentage of probability $(P=$ release/demands $\times 100)$. Between nose-poking and food delivery, a chamber light was turned on for $4 \mathrm{~s}$. The difference between demands for certain vs. uncertain reward was signaled by turning on the chamber light over the feeding or non-feeding magazine, respectively. Following released (or omitted) food delivery, the light inside the feeding (or the non-feeding) magazine was turned on for $15 \mathrm{~s}$, during which nose-poking was recorded but was without scheduled consequences (time-out). Hence, animals had to make a choice between a SS or a large/lucklinked (LLL) reward. For large-reward delivery, the $P$ level was fixed for each daily session, and was diminished progressively over subsequent days. The small-reward delivery was unchanged.

Probability $P$ values can be divided into two distinct fields, separated by the indifferent point, namely the $P$ value at which choosing either hole would yield an identical food gain. This value, calculated as "small reward size"/"big reward size," is $20 \%$ in our work, in that demanding five pellets delivered $20 \%$ of times is theoretically indifferent from demanding one pellet. In the training phase (at least 1 week), whereby we used a range of $P$ values before the indifferent point $(100 \%>P>20 \%)$, the risk of losing large reinforcement is mild relative to its size. Under these conditions, driven by "economical convenience," rats continue to prefer choosing for LLL, as we have shown previously (Adriani and Laviola, 2006). In the test phase (1 week), whereby we used a range of $P$ values beyond the indifferent point $(20 \%>P>0 \%)$, it would be "economically convenient" for rats to choose for SS (the average outcome of LLL choice being less than one pellet per nose-poking). Under these conditions, however, animals may be tempted to gamble for the probabilistic reward, rather than shift to the certain one. Indeed, the "lucky event" of food delivery would be quite rare but lead to binge reinforcement. This kind of "instinctive temptation" may overcome other decision criteria, and take control of behavior despite a diminished overall food gain. 
Experiment 4: Quantitative real-time PCR, Western blotting

At least 1 week after the end of behavioral analyses, the DAT+ and GFP groups were selected for neurochemical analyses. This selection was done since animals inoculated with Lenti-DATsiRNAs display $>90 \%$ silencing of endogenous DAT expression (Boyer and Dreyer, 2008) and are also not doxycycline-regulatable. Half of the rats were given doxycycline $(0.02 \%$ in tap water) for a Lenti-DAT switch off, the other half were given tap water (to keep Lenti-DAT still on) for 2 days. These two conditions were counterbalanced across subgroups detected in operant behavior. At sacrifice, brains were quickly removed on ice. One whole brain per group was frozen and used for a histological verification of the injection site (data not shown). From other brains, NAcc tissues were dissected out bilaterally. Tissues from either hemisphere were randomly assigned for DAT mRNA or protein analysis.

For quantitative real-time PCR, primer sets for rat DAT were designed to amplify 100- to 200-bp products, using the PRIMER3 software (http://frodo.wi.mit.edu/cgi-bin/primer3/primer3). The following specific primer pairs were used for rat DAT: $5^{\prime}$-GTT CTA CGG CGT CCA GCA-3' and 5'-TGA CCA CGA CCA CAT ACA GG-3'. Total RNA was extracted from NAcc tissue using TRIzol Reagent (Invitrogen, Basel, Switzerland), including an RNase-free DNase step. RNA was then quantified by spectrophotometry, and its integrity verified by agarose gel electrophoresis as visualized with ethidium bromide staining. First strand CDNA was generated from $2 \mu \mathrm{g}$ of total RNA and Oligo( $\left(\mathrm{dT}_{12-18}\right)$-primer with the M-MLV reverse transcription kit (Invitrogen) in a total volume of $20 \mu \mathrm{l}$. The reaction product was used for quantitative real-time PCR using the real-time PCR iCycler (BioRad, Reinach, Switzerland). Two microliters of cDNA preparation, $0.5 \mu \mathrm{M}$ of forward and reverse primers and $10 \mu \mathrm{l}$ of IQ SYBR Green Supermix (Biorad) in a total volume of $20 \mu \mathrm{l}$ were applied and PCR was performed as follows: $3 \mathrm{~min}$ at $95^{\circ} \mathrm{C}$ (initial denaturation); $20^{\circ} \mathrm{C} / \mathrm{s}$ temperature transition rate up to $95{ }^{\circ} \mathrm{C}$ for $45 \mathrm{~s}, 45 \mathrm{~s}$ at $62{ }^{\circ} \mathrm{C}$, repeated for 40 cycles (amplification). The PCR reaction was evaluated by melting curve analysis and by checking the PCR products on $2 \%$ agarose gel. The PCR cycle number at which each assay target reached the threshold detection line was determined ("threshold cycles," Ct value). The $\mathrm{Ct}$ of each candidate was normalized against that of cyclophilin F, used as an internal control (Bahi et al., 2004a,b). To determine the linearity and detection limit of the assay, cDNA samples were amplified for successive 10 -fold dilutions in a series of real-time PCRs, using duplicate assay on each dilution, so that the correlation coefficient could be calculated from the standard curve of $\mathrm{Ct}$ values. The $\Delta \mathrm{Ct}$ for each candidate was calculated as: $\Delta \mathrm{Ct}=[\mathrm{Ct}$ (candidate) $-\mathrm{Ct}$ (cyclophilin F)]. The relative abundance of each target can be calculated as the ratio between the target and reference cyclophilin $\mathrm{F}$ levels (Bahi et al., 2004a,b).

For Western blotting, samples of NAcc tissue (150-200 mg) were homogenized in buffer $(50 \mathrm{mM}$ Tris $\mathrm{pH} 7.5,120 \mathrm{mM} \mathrm{NaCl}$, $1.5 \mathrm{mM} \mathrm{CaCl}_{2}, 5 \mathrm{mM} \mathrm{MgCl}_{2}, 5 \mathrm{mM} \mathrm{KCl}, 5 \mathrm{mM}$ EDTA) with a protease inhibitor mixture (Sigma, St. Louis, MO, USA; $1 \mathrm{~mL} / 20 \mathrm{~g}$ of tissue). Homogenates were solubilized with $1 \%$ digitonin, followed by the addition of secondary solubilization buffer $(50 \mathrm{mM}$ Tris, $\mathrm{pH} 7.6,150 \mathrm{mM} \mathrm{NaCl}, 1 \%$ Nonidet-P40, $0.5 \%$ sodium deoxycholate, $2 \mathrm{mM}$ EDTA, $1 \mathrm{mM}$ sodium ortho-vanadate, $1 \mathrm{mM}$ PMSF, and $1 \%$ Triton $X-100$ ), and centrifuged at $10,000 \times g$ at $4{ }^{\circ} \mathrm{C}$ for $15 \mathrm{~min}$. Solubilized extracts were subjected to SDS-PAGE. Blots were blocked with $5 \%$ nonfat dried milk dissolved in TBST (10 mM Tris, $150 \mathrm{mM} \mathrm{NaCl}$, and $0.1 \%$ Tween-20) for $1 \mathrm{~h}$ at room temperature and incubated with primary antibodies for DAT (1:2000; Abcam, UK) overnight at $4{ }^{\circ} \mathrm{C}$. Blots were washed three times with TBST and incubated for $1 \mathrm{~h}$ with the peroxidaseconjugated secondary antibody solution. Proteins were then visualized using enhanced chemi-luminescence (Millipore, Basel, Switzerland). Membranes were washed for $30 \mathrm{~min}$ in TBST and placed in stripping solution (glycine- $\mathrm{HCl} 25 \mathrm{mM}, \mathrm{pH} 2.0,1 \%$ SDS) for $30 \mathrm{~min}$ and used as described above for detection of $\beta$-actin (1:4000; Sigma), used as an internal control. All signals from the blots were estimated using a multi-analyst software.

\section{Design and data analysis}

Behavioral analyses. Data were analyzed by multifactorial ANOVA. The general design of all experiments was a four-level group factor (GFP, DAT, SIL, NOP) $\times$ repeated measures on the same subject. As a whole, the NOP group never differed from the control GFP group in all behavioral tests, and thus data from this group are not shown. For the novelty-preference paradigm (Exp. 1), a two-level side (familiar vs. novel) and a time (5-min time points) factor were added. For the impulsivity paradigm (Exp. 2), a delay factor $(0-75 \mathrm{~s})$ was added. For the probabilistic-delivery paradigm (Exp. 3), a probability factor $(20 \%$ to $6 \%)$ was added. Multiple comparisons were performed when allowed with Tukey HSD test.

Correlation analysis. Data on impulsivity and probabilisticdelivery were formally studied for potential correlation. Specifically, for each experimental rat, the slope of the intolerance-todelay and of the probabilistic-delivery curves was calculated using Microsoft Excel "slope" function. The slope values of the two curves, coupled for each individual rat, were analyzed using the Pearson linear correlation.

Neurochemical analyses. For Western blot quantification and for real-time PCR, the analysis comprised the DAT group and GFP controls, under both doxycycline regimens. ANOVA design used the regimen (tap water vs. doxycycline) as a within-subject factor and the lentiviral inoculation as the between-subjects factor. The statistical significance was set at $P<0.05$.

\section{RESULTS}

\section{Experiment 1: Novelty-preference task}

Activity in response to forced and free-choice novelty. The ANOVA for activity rate expressed on the first day of exposure to a novel environment yielded no main effect or interaction of the group factor. Thus the four groups did not differ for this parameter. The ANOVA for crossing between compartments expressed on the test day yielded no main effect or interaction of the group factor. Thus the four groups did not differ for this parameter.

Novelty preference. The ANOVA for time spent in the novel side yielded a main effect of group, $F(3,35)=2.35$, $P<0.05$, and a tendency for group $\times$ time interaction, $F(21,245)=1.41, P<0.10$. Using the minimal significant difference, calculated by Tukey HSD test, we determined whether groups spent significantly more time in the novel side compared with chance level. We found that the GFP controls significantly preferred the novel side at all time points, as expected, with a peak at the 30 and 35 min time points. Conversely, both DAT and SIL rats had no significant preference for novelty and appeared to explore both sides at chance level, except for the last time point (see Fig. 1). Post hoc comparisons among groups revealed that, compared with GFP controls, both DAT and SIL groups explored the novel side significantly less at the 30 and 35 min time points. Moreover, the SIL group showed significantly less exploration of the novel side during the first 5 min after door opening. 


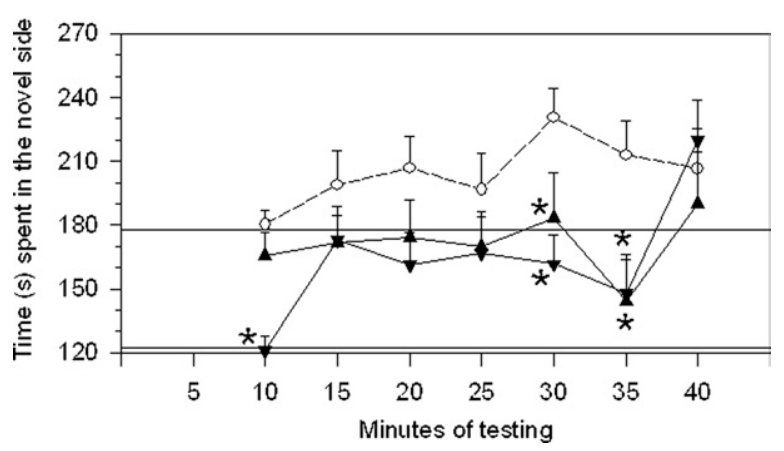

Fig. 1. Novelty preference. Time (s) spent in the novel side of a two-chamber apparatus during 5-min intervals. Rats were previously familiarized to one side. On testing day, the DAT+ (upward triangle) and SIL (downward triangle) rats were apparently not preferring the novel over the familiar side, as was evident for controls (open circle). The lines denote the chance level as calculated by $50 \%$ (i.e. 150s) plus/minus the minimal statistical difference from the Tukey HSD. * $P<0.05$ compared with GFP controls ( $n=10$ per group).

In summary these findings demonstrated a suppression of novelty seeking within DAT over-expressing and the DAT silencing rats, with no effects whatsoever on locomotor reactivity to both forced and free-choice novelty.

\section{Experiment 2: Delay-intolerance task for impulsivity}

Few animals (around one per group) were excluded due to failure to perform a task correctly (criteria for exclusion: a preference for LL less than $50 \%$ at delay $=0$ and/or more than $50 \%$ at delay $=75 \mathrm{~s}$ ). The ANOVA for LL preference revealed a main effect of delay, $F(7,224)=144.6, P<$ 0.001 , indicating the expected shift from LL to $S S$ as a function of increasing delays, and of group, $F(3,32)=2.41$, $P<0.05$, suggesting that viral inoculation was able to affect impulsivity. Two separate ANOVAs were performed on the first and second half of the curve (four sessions each). Unpublished work from our group suggests indeed that a peak in behavioral flexibility occurs between $30 \mathrm{~s}$ and $45 \mathrm{~s}$ of delay. The delay by group interaction did not appear for the first half but was significant, $F(9,96)=2.56, P<0.05$, for the second half. The multiple comparisons for the first half revealed that DAT + and SIL curves were lower than control at $10 \mathrm{~s}$ and $20 \mathrm{~s}$. The multiple comparisons for the second half revealed that only the DAT+ curve was still lower than control at $30 \mathrm{~s}$ and at $45 \mathrm{~s}$. At $75 \mathrm{~s}$ the GFP control was higher than all other groups (see Fig. 2).

As for "inadequate responding," nose-poking in either hole during timeout periods and/or the course of the delay interval provides an index of "motor impulsivity," i.e. the inability to inhibit an unnecessary response (Sagvolden and Sergeant, 1998). This measure, however, yielded no significant effects across groups (data not shown), thus supporting the "cognitive" nature of impulsivity shown by DAT+ rats (Evenden, 1999).

Hence, the DAT over-expressing rats, and the SIL ones to a lesser extent, were more impulsive than controls. Noteworthy, this robust finding was obtained without the need of splitting the groups into halves, i.e. the most and least impulsive subgroups (see Adriani et al., 2003). Without such splitting, all the variability due to the presence of individual differences is loaded to the residual error, instead of the sub-population factor, and the ANOVA is hence much more conservative. In these conditions, only stronger effects can be detected, thus we may conclude that impulsivity generated by DAT enhancement was a robust phenomenon.

\section{Experiment 3: Probabilistic-delivery task for risk proneness}

After the 1-week training with $P$ values set before the indifferent point (i.e. $100 \%, 66 \%, 50 \%, 33 \%, 20 \%$ ), all rats were able to perform the task correctly and to maintain a significant preference for the large reward (i.e. a preference for LLL more than $50 \%$ at $P=20 \%$. Animals were then tested with $P$ values well beyond the indifferent point (i.e. $20 \%, 14 \%, 10 \%, 8 \%, 6 \%$ ), to assess proneness to take risks in order to get the larger reward.

Interestingly, elevated inter-individual variability appeared in all groups, with huge standard errors, suggesting that distinct sub-populations may exist (Piazza et al., 1991), thus increasing the risk of missing to detect group differences. This variability is also shown by slope values (Fig. 4). In the impulsivity task, slopes range between -20 and -40 . Conversely, in the probabilistic task, they range from 0 to -80 , thus preventing the detection of significant group effects. All groups were thus divided into two subgroups, based on the median slope value (Adriani et al., 2003). The general ANOVA yielded significance for the subgroup by probability, $F(4,112)=11.8, P<0.001$, and for

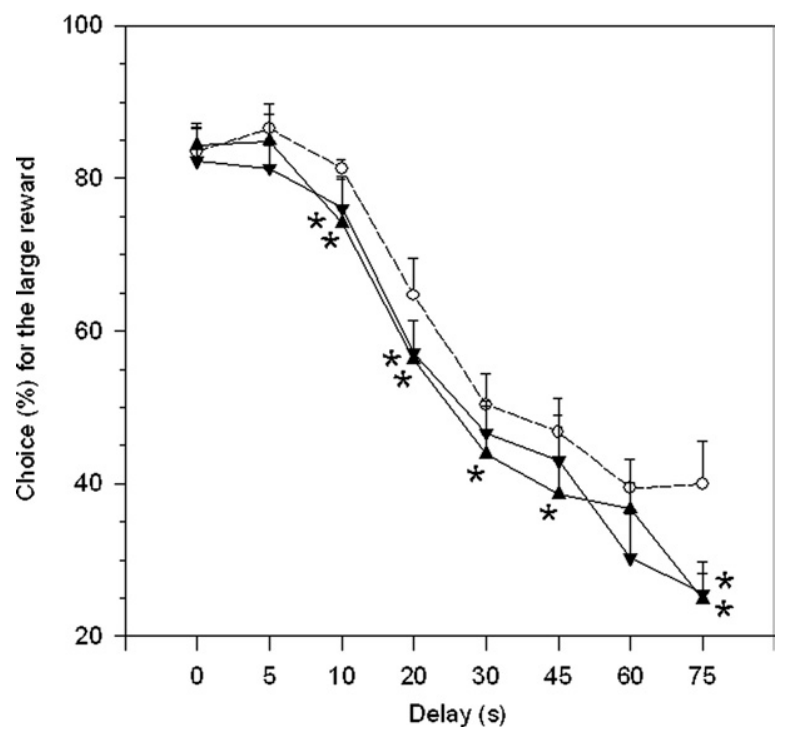

Fig. 2. Impulsive aversion. Choice (\%) for the large but delayed reward in a delay-intolerance task. Rats had a choice between one food pellet delivered immediately, or five food pellets delivered with a given delay that increased progressively. The DAT + (upward triangle) and SIL (downward triangle) rats were more impulsive than controls (open circle). * $P<0.05$ compared with GFP controls $(n=8 / 10$ per group). 
Table 1. Choice (\%) for the large but uncertain reward in the "conservative" sub-population

\begin{tabular}{|c|c|c|c|c|c|}
\hline Group & $P=20 \%$ & $P=14 \%$ & $P=10 \%$ & $P=8 \%$ & $P=6 \%$ \\
\hline GFP control & $65.4 \pm 5.7$ & $57.8 \pm 5.7$ & $45.6 \pm 5.9$ & $47.0 \pm 6.2$ & $31.1 \pm 1.7$ \\
\hline $\mathrm{DAT}+$ & $64.7 \pm 3.6$ & $53.5 \pm 5.5$ & $48.6 \pm 5.3$ & $42.5 \pm 7.3$ & $31.5 \pm 3.7$ \\
\hline DAT - & $68.7 \pm 7.1$ & $58.2 \pm 10.7$ & $47.1 \pm 9.3$ & $46.3 \pm 8.6$ & $29.6 \pm 7.7$ \\
\hline NOP & $61.3 \pm 4.9$ & $59.7 \pm 2.3$ & $46.0 \pm 5.4$ & $43.4 \pm 4.3$ & $25.1 \pm 4.5$ \\
\hline
\end{tabular}

In a probabilistic-delivery task, rats had choice between one food pellet delivered for sure (SS), or five food pellets delivered with a given probability that decreased progressively (LLL). Here we show the animal subgroup which shifted quickly to SS as probability decreased ( $n=5$ per group). The other subgroup, showing nearly no shift from LLL to SS, is shown in Fig. 3.

the group by subgroup by probability, $F(4,112)=1.72$, $P<0.05$, interactions. As a general rule across the four groups, half of the animals shifted quickly to SS (i.e. they displayed a "conservative" strategy), while half of them showed nearly no shift despite the decreased level of LLL probability (i.e. they displayed a "risk prone" strategy). Thus, the median slope value (in the probabilistic task) allowed us to discriminate between the "conservative" and "risk prone" rats.

Two separate ANOVAs were performed within subgroups. Within the "conservative" (i.e. the SS-shifting) subgroup, all the four curves were very close to each other and no group effect appeared (see Table 1). As a whole, these "conservative" rats do shift from an LLL preference to a clear SS preference, and this shift is observed for $P$ values between $14 \%$ and $10 \%$. By the end of the task, all animals displayed a clear-cut preference for the SS option, with SS choices becoming at least twice than LLL choices. Conversely, within the "risk prone" (i.e. the non-shifting) subgroup, the overall profiles were clearly different (see Fig. 3). These rats never clearly abandoned the LLL choices, and remained above the indifference point of $50 \%$ at least until $P=8 \%$. Then, controls and SIL rats eventually showed a shift, but only at the lowest $P$ value. Interestingly, DAT+ rats did not shift at all, thus suggesting a proneness to take risks (see Discussion). Such descriptive features suggest a simple criterion for identifying the two subgroups in future studies. This criterion may consist in animals reaching (or not reaching) a clear-cut preference for SS, namely an LLL preference lower (or higher) than 33\% (respectively) at the end of the task.

The "risk prone" (i.e. non-shifting) subgroup of rats was of course most interesting. Among them, the ANOVA for LLL preference revealed a main effect of probability, $F(4,60)=12.36, P<0.001$, indicating that there was indeed a significant shift from LLL to SS as a function of decreasing probability, although this was small in magnitude. Interestingly, the ANOVA revealed significance for group, $F(3,15)=2.55, P<0.05$, and a clear tendency for the group by probability interaction, $F(12,60)=1.63$, $0.10<P<0.05$. Multiple comparisons revealed that DAT over-expressing rats' curve was higher than control at all the tested $P$ values, with the exception of the starting point $P=20 \%$. Hence, a risk proneness was elicited by rarefaction of successful LLL delivery, specifically in some of the DAT+ rats.

\section{Correlation between impulsivity and risk proneness curves}

With the aim of searching for potential correlation between the slope values of intolerance-to-delay and probabilisticdelivery curves, the Pearson's $R$ value was calculated for the whole batch of animals and then independently for each group. These two parameters showed no correlation when considering the whole batch of rats $(R=-0.079$, NS for $N=40)$, or when we decided to consider only the control and the DAT + $(\mathrm{R}=-0.335$, NS for $N=20)$ groups. Intriguingly, when considering the groups separately, a tendency toward a significant correlation was found for the DAT over-expressing group, with impulsivity being directly correlated to risk-proneness slope $(R=-0.510 ; 0.10<P<0.05$ for $N=10)$. Namely, flatter values of the probabilistic-delivery slope tended to be associated with steeper slopes in the intolerance-to-delay curve. In contrast, no correlation was again found within controls $(R=-0.028$, NS for $N=9)$

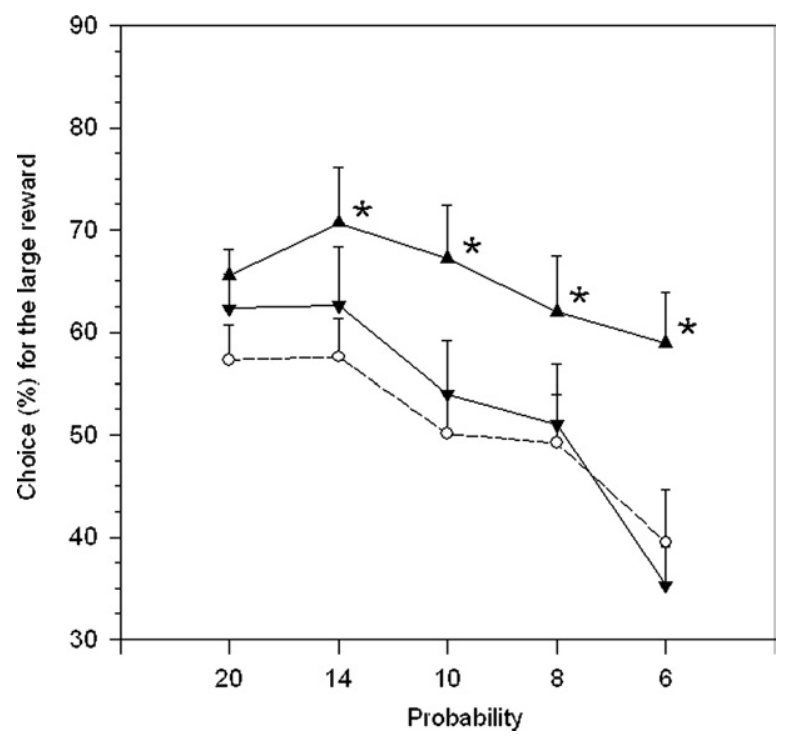

Fig. 3. Risk proneness. Choice (\%) for the large but uncertain reward in a probabilistic-delivery task, shown by the "risk prone" sub-population (see Experimental Procedures). Rats had a choice between one food pellet delivered for sure, or five food pellets delivered with a given probability $P$ that decreased progressively. The other "conservative" sub-population is shown in Table 1. The DAT + (upward triangles) rats were more prone to take risks, as shown by their stable LLL preference, than control (open circles) and SIL (downward triangles) ones. NOP and GFP control rats did not differ significantly. * $P<0.05$ compared with GFP controls ( $n=5$ per group). 
Correlation within DAT+

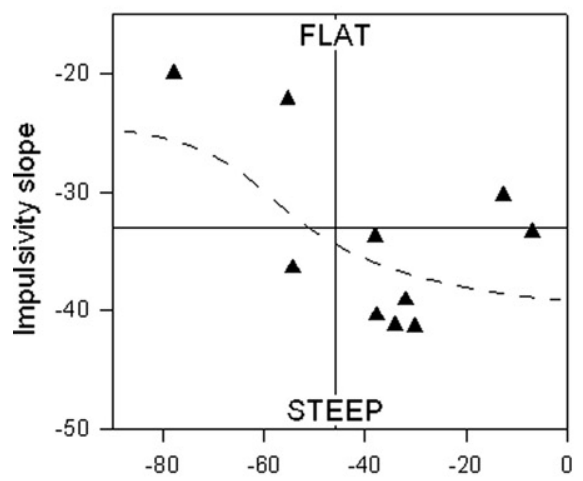

Correlation within GFP

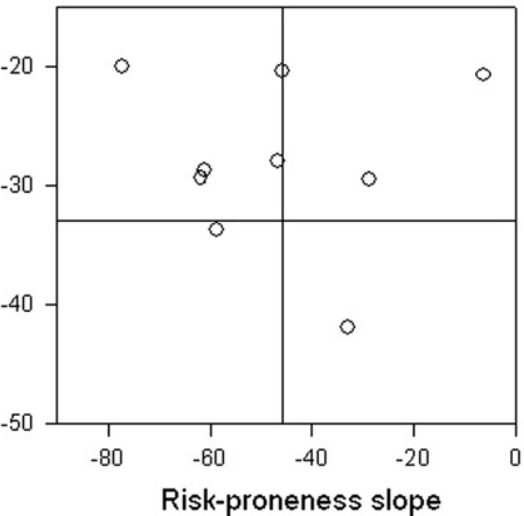

Correlation within SIL

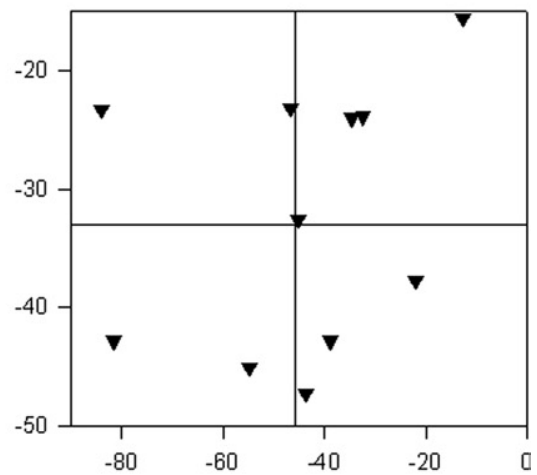

Fig. 4. Correlation between impulsivity and risk proneness. For each rat, the slope of the delay-intolerance and of the probabilistic-delivery curves was calculated using the Microsoft Excel "slope" function. Each individual rat of the DAT+ (left panel), GFP control (middle panel) and SIL (right panel) groups is represented by a point, with slopes of these two curves as coordinates. These two slope values then underwent a Pearson linear correlation. The dotted curve in the right panel delineates the two "steep" and "flat" subgroups (see Results).

or within SIL rats $(r=0.166$, NS for $N=10)$, thus underlining the specificity of a DAT-enhancement effect.

The cloud of individual points (see Fig. 4) was rather widespread for SIL group. In the control group, a tendency to occupy the upper left quadrant appeared. Interestingly, the cloud of DAT + rats tended to occupy the lower right quadrant of the correlation graph, thus confirming increased tendency to impulsivity and risk proneness. Also, $\mathrm{DAT}+$ rats appeared to segregate into two clusters, which correspond to the same sub-populations previously identified (Adriani et al., 2003). Indeed, by splitting the entire DAT + group into halves, based on the median value of impulsivity slope, we could easily identify: 1) a cluster of "steep" individuals, whose slope ranged around $-35 /-40$ for both intolerance-to-delay and probabilistic-delivery tasks; 2) a cluster of "flat" individuals with more extreme traits, whose slope was only around -20 for either intolerance-to-delay or probabilistic-delivery tasks. When the correlation was run again for these two subgroups separately, it reached the strongest level $(R=-0.86$ and -0.98 for "steep" and "flat" subgroups, respectively, $P<0.05$ for $N=5$ ), confirming the specificity of a DAT-enhancement effect.

As a matter of fact, the clouds of both "flat" and "steep" DAT + subgroups were inclined from the upper left to the lower right quadrant (see Fig. 4), indicating a clear-cut relationship between temptation by uncertainty and aversion to delay, generated within DAT + rats. This piece of data suggests that, whereas aversion to delays and conversely attraction for an uncertain-but-big reinforcer were independent phenomena in control and SIL rats, they were strongly associated following enhanced accumbal DAT expression. Thus, the production of an "impulsive and risk prone" phenotype in DAT + rats seems a specific phenomenon, somewhat reminiscent of a poorer self-control capacity. The topic certainly deserves further and deeper investigation.

\section{Experiment 4: DAT mRNA expression and DAT protein expression}

When animals had been sacrificed in the absence of doxycycline, induction of DAT mRNA expression was observed in rats inoculated with Lenti-DAT, with a ratio of $\sim 1.5$ compared with Lenti-GFP inoculated controls (Fig. 5). When animals had been sacrificed upon doxycycline regimen, the DAT expression dropped, and reached levels very similar to those observed for GFP-inoculated animals, $F(3,16)=38.9, P<0.01$. Beta-actin mRNA expression was not affected: ratios between 0.4 and 0.5 were found for all groups under all regimens of doxycycline.

In the absence of doxycycline, rats inoculated with Lenti-DAT displayed a significant increase of DAT protein levels, compared with Lenti-GFP-inoculated ones (Fig. 6). In presence of doxycycline, the DAT expression was reduced to levels very similar to those observed in the control group, $F(3,32)=23.35, P<0.01$. These findings confirm the enhanced DAT levels in DAT+ rats.

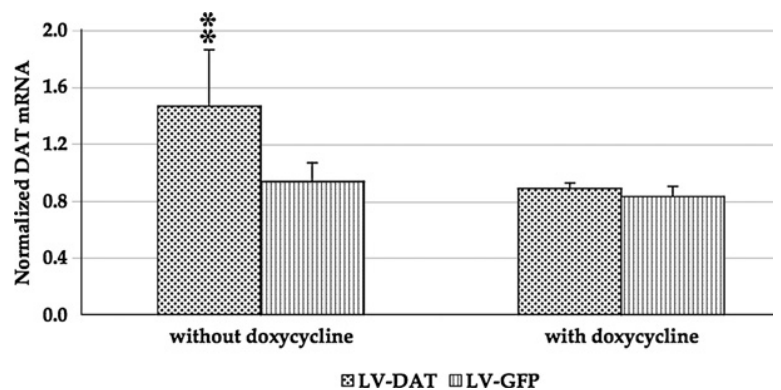

Fig. 5. qRT-PCR quantifications of DAT mRNA expression in the NAcc. At the end of the behavioral tests, animals were sacrificed and NAcc was dissected out. Total RNA was extracted, mRNA expression levels were measured by quantitative real time-PCR after reverse transcription. Quantitative mRNA levels were normalized against cyclophilin $\mathrm{F}$. The $\beta$-actin mRNA was also analyzed as endogenous control. ** $P<0.01$, compared with DAT detected from LV-GFP control rats ( $n=4$ per group) 


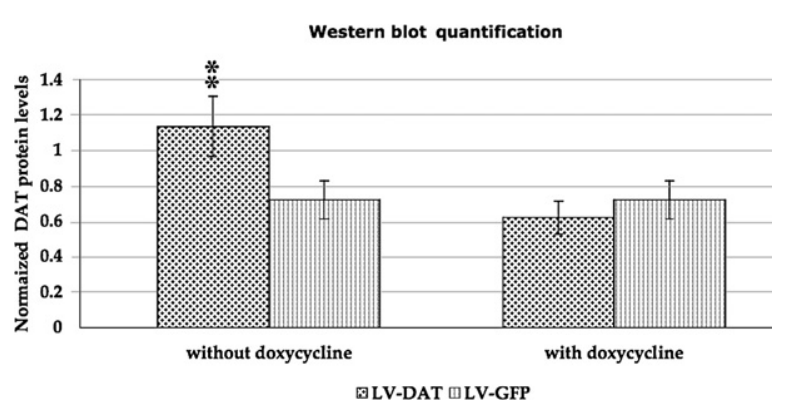

Fig. 6. Western blot analysis of DAT protein expression in the NAcc. At the end of the behavioral tests, animals were sacrificed and NAcc was dissected out. DAT signals were estimated from the blots using multi-analyst software and normalized against $\beta$-actin signal, thus providing a semi-quantitative quantification of DAT protein. ${ }^{* *} P<0.01$, compared with DAT detected from GFP control rats ( $n=4$ per group).

\section{DISCUSSION}

Decisions in everyday life often involve a balance between anticipated reward value and adverse components, such as work needed to complete, waiting requirement before delivery, or potential risk of negative unforeseen outcomes. Decision making refers to a process requiring evaluation among alternatives, which involves a motivational conflict between "optimality" of a strategy and its "affective" cost (Adriani and Laviola, 2008) as well as a continuous refinement by feedback learning from actual outcomes (Cardinal et al., 2004; Christakou et al., 2004).

\section{Impulsivity and novelty seeking}

Alterations of the dopaminergic tone were able to affect the motivation for novelty in the present rats. Both the noveltyseeking (NS) and the intolerance-to-delay (ID) tasks are probing the central DA pathways. Indeed, mammals are spontaneously motivated to search for and to explore novel stimuli (Zuckerman and Kuhlman, 2000; Bardo et al., 1996), an experience also activating the meso-limbic dopaminergic system (Rebec et al., 1997a,b). Impulsivity paradigm assumes that an instinctive aversion is generated when reward is unexpectedly delayed. Such potent and innate drives toward delay intolerance may arise within the dopaminergic meso-limbic circuits: indeed, the NAcc is known to account for the affective evaluation of reward features (Cardinal et al., 2004; Christakou et al., 2004) and plays a pivotal role in setting the maximal sustainable effort toward goals (Salamone and Correa, 2002). In this physiological frame, the NAcc might eventually support aversion for late reinforcement, especially when the waiting times are perceived as becoming excessive (SonugaBarke, 2005).

These peculiar novelty-seeking and impulsive traits are transiently expressed during the phase of adolescence (Arnett, 1992; Laviola et al., 2003), but in some vulnerable individuals can then be maintained at adult ages, leading to extreme (borderline, if not pathological) behaviors like e.g. drug misuse, exaggerate sexual activity, reckless driving, and also PG (Zuckerman and Kuhlman, 2000). Psycho-genetic investigation has shown a substantial genetic influence in these traits, although the interaction with en- vironmental factors shall be considered (Caspi et al., 2003). The DA D4 receptor gene is the prototypic polymorphic component of the background for novelty-seeking (Ebstein et al., 1996), PG (Comings et al., 1999), and ADHD (Faraone et al., 2005). Other polymorphic genes of monoamine systems (like e.g. DAT) are still under investigation. Present data suggest that disturbance within the DA system, in either direction, reflected in a loss of curiosity-driven exploration and in a lack of inhibitory selfcontrol feedback. The suppression of novelty seeking came with no effects whatsoever on locomotor reactivity to both forced and free-choice novelty. Presently, basal levels of spontaneous locomotion have not been assessed. However, in a replication study we recently performed, rats underwent the same DAT manipulation but different behavioral endpoints. Again, no differences in locomotor activity were found in social-interaction and anxiety tests (Adriani et al., manuscript in preparation).

These data are highly consistent with literature, reporting that either stimulation or blockade of DA receptors leads animals to be less motivated for novelty and more impulsive than controls (Bardo et al., 1993; Evenden and Ryan, 1996; Wade et al., 2000). The literature on psychostimulant drugs, however, is mixed (Seeman and Madras, 1998). For instance, opposite effects are found for amphetamine in the delay task (Evenden and Ryan, 1996; Wade et al., 2000). It is now accepted that these paradoxical findings can be due to differences in experimental procedures (Cardinal et al., 2000), or particularly to a baselinedependent effect (Barbelivien et al., 2008). In our work, where the delay interval was signaled, increased choice for the large reinforcer could be expected for SIL rats, like it would be expected with amphetamine (Cardinal et al., 2000) and methylphenidate (Adriani et al., 2004). However, the slightly enhanced impulsivity of SIL rats shall be discussed in terms of the reduced motivation also found in the novelty seeking test. It is likely that this group was not motivated enough to sustain the value of the larger prize across its late delivery. Such an explanation would not tap onto instinctive, delay-induced aversion but directly on perception of reward magnitude. These animals were not simply governed by a hyper-dopaminergic state, as compensating feedback reactions may have occurred. These results agree with authors reporting that changes in DA tone, by influencing cognitive control and motivation, may be strongly implicated in pathogenesis of ADHD (Schultz et al., 1997; Casey and Durton, 2006) and depression (Chau et al., 2004).

\section{Impulsivity and risk proneness}

The over-expression but not silencing of accumbal DAT leads animals to be much more attracted by a binge-butrare reward. Apparently, in such kind of probabilistic task, not all subjects reacted in the same way. Indeed, we identified one subset of rats that quickly shifted to SS beyond the indifferent $(P=20 \%)$ point, and one subset that did not. Such individual differences were found in all groups, clearly indicating a phenomenon that was independent from accumbal DAT manipulation. In other words, 
when facing a progressive rarefaction of successful LLL delivery, nearly half of all animals shifted their preference to the small reinforcer, since it was scheduled to come for sure. An aversion for excessively uncertain reinforcement can be supported by NAcc due to its physiological role (Cardinal and Howes, 2005). Moreover, DAT expression level in itself had no effect on the quickly shifting subgroup, suggesting DAT plays little or no role with respect to the developing of preference for the more conservative rewarding strategy (Cardinal et al., 2002). However, the rarefaction of reinforcement offers the opportunity to elicit some features of gambling behavior when $P$ levels are set well beyond the indifferent point (Adriani and Laviola, 2006).

In the present work, indeed, we found that rarefaction in successful delivery of the large reward did not induce a shift from LLL to SS in some DAT + rats which were also among the most impulsive. Such a finding may be interpreted in several ways: either "perseverance" or "lack of value adjustment" could be proposed in DAT+ rats, and both would be consistent with the known roles of NAcc (Cardinal et al., 2004; Christakou et al., 2004; Salamone and Correa, 2002). However, the same DAT+ rats did not show LL perseveration in the impulsivity task, and were fully able to devaluate LL when its delayed delivery consistently generated an aversion to it (present data). Thus, we rather propose that DAT + rats were less able to consider the risks (i.e. reduced overall payoff) associated with large-reward rarefaction. In other words, enhanced accumbal DAT elicited a risk proneness, thus suggesting an association between delay aversion and attraction for big uncertain prizes. To the best of our knowledge, this is the first report of a same subgroup of animals (with increased expression of accumbal DAT) showing both: 1) spontaneous aversion for a large reward, when its delivery is delayed, and 2) enhanced tendency to choose for (or to gamble over) a similarly large reward, when its delivery is uncertain and rare (see Adriani and Laviola, 2006). This piece of data suggests that higher accumbal DAT expression, possibly leading to lower levels of accumbal DA, may lead to disinhibited responding when dealing with rare-butbinge rewards. This intriguing topic clearly deserves more experimental investigation.

Furthermore, it is worth emphasizing that DAT overexpressing rats are, at the same time, less prone to afford delays, and increasingly attracted by uncertain-but-big reinforcers. As a matter of fact, the same "risk prone" rats, i.e. those who continued to choose for LLL even at rarest probabilities, were also the most "impulsive" subjects, i.e. those subjects who quickly shifted to SS already at the first delays. These data demonstrate for DAT+ group a large reduction of self-control capacity. Such deficiency did not allow these rats to overcome their drives, i.e. aversion to delay or temptation by uncertainty in either task. In both cases, a certain degree of inhibitory control would be useful for rats to adopt the most fruitful strategy. Namely, they would be required to better estimate which choice option is leading to a better payoff in the long term, thus leading to the decision of affording the delay constraints, and con- versely avoiding the attractiveness of rare and risky reinforcers. DAT over-expressing rats rather seem to adopt the worst of the possible strategies, possibly under the pressure of an overwhelming sub-cortical drive. More specifically, NAcc DAT over-expression was associated with an apparent reduction in the self-control capacity, as a major behavioral symptom.

Our study supports a relationship between traits of impulsivity and risk proneness, both being marked in the DAT over-expressing group, compared with control and SIL rats. Such a finding is consistent with recent work on the role of brain reward circuitry in PG, ADHD, as well as other psychiatric disorders like substance abuse and major depression (Chau et al., 2004), and is highly consistent with other research, supporting the role of impulsivity in mediating the severity of gambling behavior (Chambers and Potenza, 2003; Steel and Blaszczynski, 1998). PG has been indeed associated to the impulsive subtype of ADHD in several clinical-epidemiology studies. Pathological gamblers have been found to discount delayed rewards much more than controls (Petry, 2001), thus demonstrating an ADHD-like phenotype. Comorbidity for PG is $35 \%$ with other impulse control disorders and $20 \%$ with ADHD (Specker et al., 1995). Consistently, patients suffering jointly from $P G$ and ADHD do exhibit a significantly lower capacity to delay gratification and reduced inhibitory control when compared with those in the PG, ADHD and control groups (Rodriguez-Jimenez et al., 2006). As for temperament, PG patients score higher on the factor accounting for novelty seeking (Nordin and Nylander, 2007), a notion which is also consistent with our findings in animal model. Taken together, these findings support the notion that severity of gambling may be mediated by a pathological disturbance of impulse control (Blaszczynski et al., 1997).

\section{Methodological considerations}

The present findings, obtained by accumbal enhancement of DAT expression which possibly produced a hypo-dopaminergic state, shall be briefly compared with data from rat accumbal lesions (Cardinal et al., 2004). The core of the NAcc promotes selection of delayed and of uncertain rewards, and its lesion produces in general a conservative, risk-averse and delay-averse, pattern of choice (Cardinal and Cheung, 2005; Cardinal and Howes, 2005; Cardinal et al., 2004). In other words, accumbal damage favors the consummation of present or easily available food (i.e. animals would segregate in the lower left quadrant of a correlation graph, due to selection of soon-and-for-sure options in operant tasks). Conversely, the fully functional NAcc appears to subserve (and/or sustain over time) the salience of rewards that are not present yet, but whose delivery is possibly perceived as either probable or imminent (Cardinal et al., 2002). However, in the context of human psycho-pathology and self-control disorders, "impulsivity" refers to a steeper delay discounting while "risk taking" refers to a greater willingness to choose unlikely rewards. Clearly, to let hypo-function of accumbal DA system account for both risk proneness and delay aversion, it 
should be agreed that the NAcc may eventually support a sub-cortical aversion for delays, at least when waiting times become overtly excessive (Sonuga-Barke, 2005; Adriani et al., 2007).

Thus, in addition to the widely recognized role of NAcc, we postulated its function to play a role for: 1) subserving increased proneness toward risk-taking behaviors, and 2) the emergence of spontaneous aversion at very long delay intervals (Adriani and Laviola, 2006; Adriani et al., 2008). Hence, we propose that accumbal DA hypo-function may lead to maladaptive behavior, subjects being more prone to gamble whenever they have the opportunity, and more likely to select immediate gratification because the delayed alternative is felt as "severely" postponed. In these respects, accumbal DA hypo-function, generated in our DAT + rat model, appears to provide a full range of selfcontrol disorders.

Existing animal models of impaired DAT function, like the DAT-KO mouse, provide useful information in that they reproduce the symptom of hyperactivity (Gainetdinov and Caron, 2001) and display impaired extinction of responses in the operant food-reinforcement tasks (Hironaka et al., 2004). However, a common feature in all knockout mice models is that the gene is absent all over the CNS and entirely across development. Conversely, the DAT manipulation was very local in our rats and only started in mature animals. Thus, the methodology exploited for the present study is not intended to provide a model for the genetic origin of the human pathology, but rather to investigate the latest possibilities of modulating brain function and behavioral symptoms in the alive animal.

\section{CONCLUSION}

Our present aim was to develop a new model, whose principal advantages are localization and inducibility. DAT over-expression remains extremely local and can be modulated at will, with a switch-off over the exogenous gene by simple doxycycline exposure. This model enables testing of the very same animal for a while, first by over-expressing DAT in a specific brain area, then shutting off overexpression in that region, then coming back to over-expression. This procedure can be repeated for months, since the virally transferred gene remains incorporated permanently and exclusively in the infected cells of the targeted brain area (Boyer and Dreyer, 2008). Knock-out rats have not been developed yet, and even KO mice by no means enable the exclusive flexibility of very local, regulatable and highly specific gene expression. The lentivirusmediated gene transfer, previously validated (Boyer and Dreyer, 2008) and presently exploited, does enable assessment of the role of specific brain nuclei in a complex behavioral pattern.

This kind of information is relevant to provide a rationale for innovative therapeutic intervention. In conclusion, our results demonstrate that the lentiviruses can reliably be used for over-expressing or silencing accumbal DAT expression, and do validate lentiviruses as a tool to study brain/behavioral function. Moreover, the observed behav- ioral changes appear to mimic, at least in part, some behavioral symptoms of ADHD and/or PG. These powerful gene-transfer tools could allow new therapeutics strategies for the treatment of cognitive and motivational diseases.

Acknowledgments-Research performed along the lines of the "ADHD-sythe" young-investigator project (to WA): "under-40 call" of Italian Ministry of Health (year 2008, assigned); "ERC-StG call" of EU-FP7 ideas (pending application). Other supporting sources: by the bilateral Italy-USA Program on Rare Diseases and by the ERARE-EuroRETT Network (to GL); by Swiss National Foundation grants (to JLD). SM was supported by a NARSAD young investigator award, LG was recipient of a CNR post-doc fellowship and is presently at Institute of Neurobiology \& Molecular Medicine, CNR, Roma, Italy. The authors wish to thank: European Mind and Metabolism Association (EMMA); I. Olivier (France), E. Romano (Italy), E. M. Marco-Lopez (Spain), for their invaluable help.

\section{REFERENCES}

Adriani W, Caprioli A, Granstrem O, Carli M, Laviola G (2003) The spontaneously hypertensive-rat as an animal model of ADHD: evidence for impulsive and non-impulsive subpopulations. Neurosci Biobehav Rev 27:639-651.

Adriani W, Rea M, Baviera M, Invernizzi W, Carli M, Ghirardi O, Caprioli A, Laviola G (2004) Acetyl-L-carnitine reduces impulsive behaviour in adolescent rats. Psychopharmacology 176:296-304.

Adriani W, Laviola G (2006) Delay aversion but preference for binge and rare rewards in two choice tasks: Implications for measuring of self-control parameters. BMC Neurosci 7:52.

Adriani W, Canese R, Podo F, Laviola G (2007) Reduced impulsive behavior and $1 \mathrm{H}$ MRS detectable metabolic brain changes in adult rats exposed to methylphenidate during adolescence. Neurotoxicol Teratol 29:116-125.

Adriani W, Laviola G (2009) Animal models and mechanisms of impulsivity during adolescence. In: Cerebro y mente, Part 3-The affective status and impulsive, motor \& motivational symptoms (Palomo T, ed), pp. 385-434. In press.

Arnett J (1992) Reckless behavior in adolescence: A developmental perspective. Dev Rev 12:339-373.

Bahi A, Boyer F, Gumy C, Kafri T, Dreyer JL (2004a) In vivo gene delivery of urokinase-type plasminogen activator with regulatable lentivirus induces behavioural changes in chronic cocaine administration. Eur J Neurosci 20:3473-3488.

Bahi A, Boyer F, Kafri T, Dreyer JL (2004b) CD81-induced behavioural changes during chronic cocaine administration: in vivo gene delivery with regulatable lentivirus. Eur J Neurosci 19:1621-1633.

Bahi A, Boyer F, Bussard G, Dreyer JL (2005a) Silencing dopamine D3-receptor in the nucleus accumbens shell in vivo induces behavioral changes in cocaine-induced hyperlocomotion. Eur J Neurosci 21:3415-3426.

Bahi A, Boyer F, Kolira M, Dreyer JL (2005b) In vivo gene silencing of CD81 by lentiviral expression of small interference RNAs suppresses cocaine-induced behavior. J Neurochem 92:1243-1255.

Bannon MJ (2005) The dopamine transporter: role in neurotoxicity and human disease. Toxicol Appl Pharmacol 204:355-360.

Barbelivien A, Billy E, Lazarus C, Kelche C, Majchrzak M (2008) Rats with different profiles of impulsive choice behavior exhibit differences in responses to caffeine and d-amphetamine and in medial prefrontal cortex 5-HT utilization. Behav Brain Res 187:273-283.

Bardo MT, Bowling SL, Robinet PM, Rowlett JK, Lacy M, Mattingly BA (1993) Role of dopamine D1 and D2 receptors in novelty-maintained place preference. Exp Clin Psychopharmacol 1:101-109.

Bardo MT, Donohew RL, Harrington NG (1996) Psychobiology of novelty seeking and drug seeking behavior. Behav Brain Res 77:23-43. 
Biederman J (1998) Attention-deficit/hyperactivity disorder: a life-span perspective. J Clin Psychiatry 59(S7):4-16.

Black DW, Moyer T (1998) Clinical features and psychiatric comorbidity of subjects with pathological gambling behavior. Psychiatr Serv 49:1434-1439.

Blaszczynski A, Steel Z, McConaghy N (1997) Impulsivity in pathological gambling: the antisocial impulsivist. Addiction 92:75-87.

Boyer F, Dreyer JL (2008) The role of gamma-synuclein in cocaineinduced behaviour in rats. Eur J Neurosci 27:2938-2951.

Cardinal RN, Robbins TW, Everitt BJ (2000) The effects of d-amphetamine, chlor-diazepoxide, alpha-flupenthixol and behavioural manipulations on choice of a signalled and unsignalled delayed reinforcement in rats. Psychopharmacology 152:362-375.

Cardinal RN, Parkinson JA, Hall J, Everitt BJ (2002) Emotion and motivation: The role of the amygdala, ventral striatum, and prefrontal cortex. Neurosci Biobehav Rev 26:321-352.

Cardinal RN, Winstanley CA, Robbins TW, Everitt BJ (2004) Limbic corticostriatal systems and delayed reinforcement. Ann N Y Acad Sci 1021:33-50

Cardinal RN, Cheung THC (2005) Nucleus accumbens core lesions retard instrumental learning and performance with delayed reinforcement in the rat. BMC Neurosci 6:9.

Cardinal RN, Howes NJ (2005) Effects of lesions of the nucleus accumbens core on choice between small certain rewards and large uncertain rewards in rats. BMC Neurosci 6:37.

Casey BJ, Durston S (2006) From behavior to cognition to the brain and back: What have we learned from functional imaging studies of attention deficit hyperactivity disorder? Am J Psychiatry 163: 957-960.

Caspi A, Sugden K, Moffitt TE, Taylor A, Craig IW, Harrington H, McClay J, Mill J, Martin J, Braithwaite A, Poulton R (2003) Influence of life stress on depression: moderation by a polymorphism in the 5-HTT gene. Science 301:386-389.

Castellanos FX, Sonuga-Barke EJ, Milham MP, Tannock R (2006) Characterizing cognition in ADHD: beyond executive dysfunction. Trends Cogn Sci 10:117-123.

Chambers RA, Potenza MN (2003) Neurodevelopment, impulsivity, and adolescent gambling. J Gambl Stud 19:53-84.

Chau DT, Roth RM, Green AI (2004) The neural circuitry of reward and its relevance to psychiatric disorders. Curr Psychiatry Rep 6: 391-399.

Christakou A, Robbins TW, Everitt BJ (2004) Prefrontal cortical-ventral striatal interactions involved in affective modulation of attentional performance: implications for corticostriatal circuit function. J Neurosci 24:773-780.

Comings DE, Gonzalez N, Wu S, Gade R, Muhleman D, Saucier G, Johnson P, Verde R, Rosenthal RJ, Lesieur HR, Rugle LJ, Miller WB, MacMurray JP (1999) Studies of the 48 bp repeat polymorphism of the DRD4 gene in impulsive, compulsive, addictive behaviors: Tourette syndrome, ADHD, pathological gambling, and substance abuse. Am J Med Genet 88:358-368.

Dougherty DD, Bonab AA, Spencer TJ, Rauch SL, Madras BK, Fischman AJ (1999) Dopamine transporter density in patients of attention deficit hyperactivity disorder. Lancet 354:2132-2133.

Doyle AE (2006) Executive functions in attention-deficit/hyperactivity disorder. J Clin Psychiatry 67:21-26.

Ebstein RP, Novick O, Umansky R, Priel B, Osher Y, Blaine D, Bennett ER, Nemanov L, Katz M, Belmaker RH (1996) Dopamine D4 receptor (D4DR) exon III polymorphism associated with the human personality trait of novelty seeking. Nat Genet 12:78-80.

Evenden JL, Ryan CN (1996) The pharmacology of impulsive behaviour in rats: the effects of drugs on response choice with varying delays of reinforcement. Psychopharmacology 128:161-170.

Evenden JL (1999) Varieties of impulsivity. Psychopharmacology 146:348-361.

Faraone SV, Perlis RH, Doyle AE, Smoller JW, Goralnick JJ, Holmgren MA, Sklar P (2005) Molecular genetics of attentiondeficit/hyperactivity disorder. Biol Psychiatry 57:1313-1323.
Fischman AJ, Bonab AA, Babich JW, Palmer EP, Alpert NM, Elmaleh DR, Callahan RJ, Barrow SA, Graham W, Meltzer PC, Hanson RN, Madras BK (1998) Rapid detection of Parkinson's disease by SPECT with altropane: a selective ligand for dopamine transporters. Synapse 29:128-141.

Gainetdinov RR, Caron MG (2001) Genetics of childhood disorders: XXIV. ADHD, part 8: hyperdopaminergic mice as an animal model of ADHD. J Am Acad Child Adolesc Psychiatry 40:380-382.

Hironaka N, Ikeda K, Sora I, Uhl GR, Niki H (2004) Food-reinforced operant behavior in dopamine transporter knockout mice: enhanced resistance to extinction. Ann N Y Acad Sci 1025:140-145.

Hollander E, Buchalter AJ, DeCaria CM (2000) Pathological gambling. Psychiatr Clin North Am 23:629-642.

Hollander E, Sood E, Pallanti S, Baldini-Rossi N, Baker B (2005) Pharmacological treatments of pathological gambling. J Gambl Stud 21:99-110.

Jucaite A, Fernell E, Halldin C, Forssberg H, Farde L (2005) Reduced midbrain dopamine transporter binding in male adolescents with attention-deficit/hyperactivity disorder: association between striatal dopamine markers and motor hyperactivity. Biol Psychiatry $57: 229-238$

Laucht M, Becker K, El-Faddagh M, Hohm E, Schmidt MH (2005) Association of the DRD4 exon III polymorphism with smoking in fifteen-year-olds: a mediating role for novelty seeking? J Am Acad Child Adolesc Psychiatry 44:477-484.

Laviola G, Macri S, Morley-Fletcher S, Adriani W (2003) Risk-taking behavior in adolescent mice: Psychobiological determinants and early epigenetic influence. Neurosci Biobehav Rev 27:19-31.

Lowengrub K, lancu I, Aizer A, Kotler M, Dannon PN (2006) Pharmacotherapy of the pathological gambling: review of new treatment modalities. Expert Rev Neurother 6:1845-1851.

Madras BK, Miller GM, Fischman AJ (2005) The dopamine transporter and attention-deficit/hyperactivity disorder. Biol Psychiatry 57: 1397-1409.

Maggos CE, Spangler R, Zhou Y, Schlussman SD, Ho A, Kreek MJ (1997) Quantitation of dopamine transporter mRNA in the rat brain: mapping, effects of "binge" cocaine administration and withdrawal. Synapse 26:55-61.

Mazei-Robinson MS, Blakely RD (2006) ADHD and the dopamine transporter: are there reasons to pay attention? Handb Exp Pharmacol 175:373-415.

Nordin C, Nylander PO (2007) Temperament and character in pathological gambling. J Gambl Stud 23:113-120.

Oades RD (1998) Frontal, temporal and lateralized brain function in children with attention-deficit hyperactivity disorder: a psychophysiological and neuropsychological viewpoint on development. Behav Brain Res 94:83-95.

Petry NM (2001) Pathological gamblers, with and without substance use disorders, discount delayed rewards at high rates. J Abnorm Psychol 110:482-487.

Piazza PV, Maccari S, Deminiere JM, Le Moal M, Mormede P, Simon H (1991) The corticosterone levels determine individual vulnerability to amphetamine self-administration. Proc Natl Acad Sci U S A 88:2088-2092.

Rebec GV, Christiansen JRC, Guerra C, Bardo MT (1997a) Regional and temporal differences in real-time dopamine efflux in the nucleus accumbens during free-choice novelty. Brain Res 776 : 61-67.

Rebec GV, Grabner CP, Johnson M, Pierce RC, Bardo MT (1997b) Transient increases in cathecolaminergic activity in medial prefrontal cortex and nucleus accumbens shell during novelty. Neuroscience 76:707-714.

Rodriguez-Jimenez R, Avila C, Jimenez-Arriero MA, Ponce G, Monasor R, Jimenez M, Aragües M, Hoenicka J, Rubio G, Palomo T (2006) Impulsivity and sustained attention in pathological gamblers: influence of childhood ADHD history. J Gambl Stud 22: $451-461$. 
Russell VA, Sagvolden T, Johansen EB (2005) Animal models of attention-deficit hyperactivity disorder. Behav Brain Funct 15:1-9.

Sagvolden T, Russell VA, Aase H, Johansen EB, Farshbaf M (2005) Rodent models of attention-deficit/hyperactivity disorder. Biol Psychiatry 57:1239-1247.

Sagvolden T, Sergeant JA (1998) Attention deficit/hyperactivity disorderfrom brain dysfunctions to behaviour. Behav Brain Res 94:1-10.

Salamone JD, Correa M (2002) Motivational views of reinforcement: implications for understanding the behavioral functions of nucleus accumbens dopamine. Behav Brain Res 137:3-25.

Schultz W, Dayan P, Montague PR (1997) A neural substrate of prediction and reward. Science 275:1593-1599.

Seeman P, Madras BK (1998) Anti-hyperactivity medication: methylphenidate and amphetamine. Mol Psychiatry 3:386-396.

Snyder R, Turgay A, Aman M, Binder C, Fisman S, Carroll A (2002) Risperidone Conduct Study Group. Effects of risperidone on conduct and disruptive behavior disorders in children with subaverage IQs. J Am Acad Child Adolesc Psychiatry 41:1026-1036.

Sonuga-Barke EJ (2005) Causal models of attention-deficit/hyperactivity disorder: from common simple deficits to multiple developmental pathways. Biol Psychiatry 57:1231-1238.

Sood ED, Pallanti S, Hollander E (2003) Diagnosis and treatment of pathologic gambling. Curr Psychiatry Rep 5:9-15.
Specker SM, Carlson GA, Christenson GA, Marcotte M (1995) Impulse control disorders and attention deficit disorder in pathological gamblers. Ann Clin Psychiatry 7:175-179.

Spencer TJ, Biederman J, Madras BK, Faraone SV, Dougherty DD, Bonab AA, Fischman AJ (2005) In vivo neuroreceptor imaging in attention-deficit/hyperactivity disorder: a focus on the dopamine transporter. Biol Psychiatry 57:1293-1300.

Steel Z, Blaszczynski A (1998) Impulsivity, personality disorders and pathological gambling severity. Addiction 93:895-905.

Szulc J, Wiznerowicz M, Sauvain MO, Trono D, Aebischer P (2006) A versatile tool for conditional gene expression and knockdown. Nat Methods 3:109-116.

Thapar A, O'Donovan M, Owen MJ (2005) The genetics of attention deficit hyperactivity disorder. Hum Mol Genet 14:R275-R282.

Wade TR, de Wit H, Richards JB (2000) Effects of dopaminergic drugs on delayed reward as a measure of impulsive behavior in rats. Psychopharmacology 150:90-101.

Willcutt EG, Doyle AE, Nigg JT, Faraone SV, Pennington BF (2005) Validity of the executive function theory of attention-deficit/hyperactivity disorder: a meta-analytic review. Biol Psychiatry 57:1336-1346.

Zuckerman M, Kuhlman DM (2000) Personality and risk-taking: Common biosocial factors. J Pers 68:999-1029. 